Monika KULESZA

Université de Varsovie

\title{
LE JEU DE L'ÉQUIVOQUE DANS FRÉDÉRIC DE SICILE DE CATHERINE BERNARD.
}

Tout est double jeu dans Frédéric de Sicile : l'auteur n'est pas un homme comme le «Privilège » le notifie, mais une femme, le héros du roman n'est pas un jeune prince comme annonce le titre, mais une princesse, la narration à la troisième personne qui devrait être neutre, trahit en fait la présence d'un auteur impliqué, engagé émotionnellement... Tout cela fait que nous avons affaire à une œuvre où la confusion et l'équivoque règnent et constituent la trame du récit. On a l'impression d'un jeu que l'auteur propose au lecteur.

Nous disposons d'une édition moderne des œuvres de Catherine Bernard grâce au travail éditorial du professeur Franco Piva qui a établi, présenté et annoté les textes de la romancière. Le premier volume contient ses romans et nouvelles, précédés d'une importante introduction sur la vie de l'auteur et sur chaque roman. Les avantages de cette édition sont indiscutables car la graphie a été modernisée, mais la ponctuation, le lexique et la structuration grammaticale ont été préservés.

Le roman est paru en 1680 avec pour auteur le «sieur Pradon», mais aussi bien l'avis «du libraire au lecteur » que les indices dans le texte confirment qu'il s'agit du roman juvénile d'auteur femme. Le libraire annonce que « l'auteur prend autant de soin de se cacher et autant de modestie que son ouvrage a des beautés » (PIVA: 67, 1993) ${ }^{1}$ et en même temps il livre des détails: il s'agit d'un « coup d'essai d'une personne de dix-sept ans », «qui n'a entendu jusqu'ici que des louanges » et qui est arrivée depuis peu de temps à Paris (67-68). La jeunesse et le peu d'expérience de l'auteur n'empêchent pas que «le style en [du roman] est pur et serré, les pensées naturelles, les expressions vives et d'un ton particulier »et, «s'il s'était glissé quelques fautes » (67), il faut les pardonner car le jeune auteur, étant habitué aux éloges, risque de se décourager si la critique est trop sévère. L'équivoque sur l'auteur est ainsi augmentée par les précisions du libraire admiratif qui dévoile la romancière en la cachant.

Frédéric de Sicile se veut être un roman historique car il fait référence à certains épisodes du Moyen Age, mais comme la mode de l'époque l'exige, l'histoire constitue avant tout un décor, un agrément pour le public et une sorte d'alibi qui permet de peindre les mœurs contemporaines sous couvert historique.

La classification du roman pose également un problème. Il possède de nombreux traits baroques mais il répond aussi à certains critères classiques. L'action se déroule dans un passé lointain, comme c'était obligé dans les romans héroïques du début du siècle. La romancière ne se soucie pas de rendre véridique le décor, la vraisemblance souffre des hasards et des rebondissements inattendus qui compliquent beaucoup l'intrigue. Certains clichés du roman baroque sont repris, tels le naufrage, la bataille maritime, le mystère qui entoure l'identité des héros, leur déguisement. Comme dans la première partie du siècle, le récit est parsemé de poèmes, lettres ou billets qui sont interceptés par le personnage qui

\footnotetext{
${ }^{1}$ Toutes les références au roman de Catherine Bernard ainsi que toutes les citations proviennent de l'édition de Franco Piva de 1993.
} 
n'aurait jamais dû les connaître. L'auteur recourt au procédé « d'écouter sans être $\mathrm{vu} »$ qui a fleuri dans L'Astrée et qui permet des retournements de situation les plus inattendus. La fin heureuse survient après de multiples aventures, bref, certains aspects du roman héroïque revivent sous la plume de $\mathrm{C}$. Bernard.

C'est surtout le déguisement que Mlle Bernard garde du roman baroque. Il a une longue tradition qui commence avec le roman grec d'Héliodore et qui fleurit dans L'Astrée. Le procédé ne disparaît pas à la fin du XVIIe siècle et nombreux sont les auteurs qui y recourent car le goût du mystère, du dépaysement et de l'extraordinaire dure toujours. Le plus souvent il s'agit du déguisement sans travestissement de sexe, mais on retrouve aussi la pratique contraire, le travestissement permettant de pousser plus loin le jeu des équivoques. Citons au moins Les Mémoires de la vie d'Henriette-Sylvie de Molière de Mme de Villedieu ${ }^{2}$ où l'héroïne fuit un mari jaloux déguisée en homme ; Histoire d'Hippolyte, comte de Douglas où l'auteur, Mme d'Aulnoy, permet à Julie d'échapper à un enlèvement sous un habit de pèlerin, ou encore Le Beau Polonais dans lequel Jean Préchac travestit son héros en femme qui vit des aventures à la cour d'Autriche. Dans L'Hérö̈ne mousquetaire le même auteur présente une femme qui, suivant les circonstances, soit garde son identité féminine soit se déguise en officier !

Catherine Bernard, tout en gardant certains traits baroques, suit les consignes de critiques et de romanciers ${ }^{3}$ qui renouvellent le genre, car ce qui plaisait en 1650 est en voie de disparaître en 1680. Frédéric de Sicile est nettement moins long que ses prédécesseurs baroques, l'intrigue est assez compliquée, mais il n'y a pas d'histoires secondaires qui ne se rattachent de très près à la trame de l'œuvre. La modernité du roman est surtout visible dans l'ambiguïté des sentiments des personnages principaux, dans la crise d'identité que vit l'héroïne et dans la peinture de l'amour en tant que sentiment déchirant.

L'intrigue de Frédéric de Sicile est fondée sur le déguisement : Mainfroi, le roi de Sicile qui n'a pas d'héritier mâle cache à la naissance le sexe de sa fille et la fait passer pour un garçon. La jeune princesse est élevée comme un jeune homme, « la chasse était son plus grand divertissement» (76), Frédéric «ne connaissait point d'autre passion que celle de régner » et $\mathrm{il}^{4}$ « fut instruit à tous les exercices qui sont à l'usage des hommes; il y excella et parut le prince le plus accompli de son temps » (73-74). Mais Frédéric sait surtout que «pour régner paisiblement sur le trône, il fallait aussi régner sur son cœur» (73). Son éducation et l'intérêt du royaume, menacé de passer entre les mains du roi de Mayorque, font que Frédéric accepte le subterfuge. Le déguisement n'est pas seulement extérieur, il concerne même le for intérieur du personnage.

Avec le temps, Frédéric vit la situation conflictuelle de plus en plus difficilement. Tant que l'amour ne s'en mêle pas, Frédéric joue son rôle sans que cela lui pose trop de problèmes : il pressent seulement les difficultés qui s'en suivront : «Parvenu à cette saison où l'amour fait tant de désordres, il commença de s'observer de plus près, sachant bien que, si cette passion était assez à craindre

\footnotetext{
${ }^{2}$ Le roman cité de Mme de Villedieu date de 1674, celui de Mme d'Aulnoy de 1690, ceux de Jean Préchac respectivement de 1681, 1677.

${ }^{3}$ Notamment de Pierre-Daniel Huet, Jean Segrais, l'abbé Du Plaisir, Mme de Villedieu (Coulet, 1992).

${ }^{4} \mathrm{Je}$ garde la forme grammaticale utilisée par Catherine Bernard.
} 
pour tout le monde, elle l'était encore plus pour lui, qui serait obligé de garder des mesures très embarrassantes avec ceux qui l'auraient rendu sensible. Il voyait tous les jours quantité de princes bien faits et, s'étant examiné, il ne se trouvait point encore de mouvements qui lui parussent suspects»(74). La conscience du problème rend Frédéric très vigilant, mais cela reste assez artificiel, car il ne sait pas encore, comment l'amour se manifeste. D'ailleurs le personnage a conscience que l'amour est un sentiment dangereux et inévitable mais en même temps, il croit que son déguisement va le protéger contre la passion.

Rien de plus faux. D'abord Frédéric devient l'objet de désir de presque toutes les femmes de la cour. Son déguisement l'expose donc au début à l'amour des autres femmes, à l'amour que par la force des choses une fille déguisée en garçon ne peut pas partager. Jouer le jeu veut dire tromper et faire vivre aux autres ce qu'on voudrait éviter à soi-même. Comme les amantes sont nombreuses, Frédéric décide de faire semblant d'aimer Yolande pour décourager toutes les autres. Vu ce qu'on lui a enseigné, il n'y a rien de mauvais dans un jeu des apparences que tous pratiquent : « étant persuadé que la galanterie sied bien à un jeune prince, il voulut bien affecter une passion dont les apparences ne peuvent donner de chagrin » (74). Le héros découvre vite que cela ne le protège pas suffisamment et que désormais faire face aux femmes amoureuses est son sort.

L'équivoque quant au jeu des apparences est d'autant plus explicite que l'auteur emploie tout le temps le pronom personnel «il» pour désigner la princesse déguisée au point que le lecteur se laisse prendre dans le piège et oublie presque le véritable sexe du personnage.

Le coup de foudre survient et le jeu de l'équivoque devient plus complexe car trois personnages sur quatre présents dans la scène le vivent en même temps. Frédéric et son ami le prince Léon envoient leurs serviteurs secourir un bateau. Ce sont les enfants du roi de Mayorque, Camille et Amaldée, qu'ils sauvent sans le savoir. Bien entendu le mystère de l'identité des naufragés est préservé et Frédéric ne saura même pas pendant un certain temps qu'ils sont frère et sœur. La jalousie doit naître avec l'amour, telle est la loi du genre. Catherine Bernard recourt au procédé typique pour le roman héroïque, celui d'une chaîne amoureuse : Léon tombe amoureux de Camille qui tombe amoureuse de Frédéric qui tombe amoureux d'Amaldée. Léon voit le regard troublé de Frédéric, le croit son rival et devient jaloux, Frédéric croit Amaldée amant de Camille et devient aussi jaloux.

A partir de ce moment Catherine Bernard change de tactique et désigne Frédéric par le pronom féminin « elle » ou par son titre au féminin « la princesse de Sicile », mais seulement quand il est question d'Amaldée et des sentiments de la jeune fille envers lui. Le genre masculin est réservé à tous les autres contextes. Cela engendre parfois une grande confusion ou une grande subtilité dans le texte et il faut relire certains passages pour bien comprendre le jeu sur les pronoms personnels : «Si elle [la princesse de Sicile] trouvait en lui le fils de l'ennemi de Mainfroi, un prince pour qui on le forçait à ce déguisement si singulier, enfin qui ne devait jamais porter la couronne de Sicile, elle trouvait en récompense un prince plein de charmes » $(93)^{5}$. Ou encore un autre exemple, quand la princesse de Sicile vient de quitter Amaldée: «Elle trouva Camille et l'amirale qui la cherchaient, s'étant liguées ensemble pour lui demander raison de la tromperie

\footnotetext{
${ }^{5}$ Pour mieux le faire voir, je mets les pronoms concernant Frédéric en italique.
} 
qu'il leur avait faite»(147). L'ambiguïté grammaticale n'est pas « une faute», mais un procédé délibéré qui traduit l'ambiguïté de la psychologie des personnages, et le premier pronom de la phrase se rapporte systématiquement au genre employé dans le passage qui précède.

Au début de la troisième partie, Frédéric est à Mayorque, emprisonné à la suite d'une bataille en mer, mais traité comme invité ou ami. Il s'avère alors qu'Amaldée doit épouser la princesse de Mantoue : «Frédéric », dit la romancière en continuant le masculin, «n'envisageait plus que le mariage d'Amaldée, et il alla rêver seul en attendant son retour ». Mais quelques lignes plus bas l'analyse des sentiments est faite au féminin : «Elle en eut un redoublement sensible par la jalousie que lui donna Amaldée; elle ne l'avait point encore connue, et cette passion, si violente dans tous les autres, n'était que douloureuse chez elle, et par conséquent beaucoup plus accablante » (129). La jalousie augmente la force de la passion amoureuse, mais Frédéric habitué à étouffer toutes ses émotions et à faire semblant ne peut pas agir spontanément et ne peut qu'être encore plus malheureux. Le déguisement est un double obstacle, car l'héroïne ne se fait pas reconnaître physiquement et surtout ne peut pas exprimer ce qu'elle ressent. Pourtant : «bien qu'elle ne parût point sous la figure d'amante, elle ne l'était pas moins pour cela » (95).

L'équivoque concerne donc les sentiments et le comportement de Frédéric qui doit jouer à l'homme et qui n'en reste pas moins femme. Parfois Frédéric ne sait peut-être pas lui-même quelle est son identité. Une personne qui a du succès auprès des représentants du sexe opposé essaye le plus souvent d'en profiter pour accumuler le plus possible de conquêtes, surtout si elle ne prend pas ses engagements au sérieux. Au début du roman Frédéric fait tout le contraire : «Cependant le prince de Sicile, voyant la facilité qu'il avait d'engager les cœurs, voulut bien les épargner » (75). La romancière explique l'attitude du personnage par son manque d'intérêt pour l'amour quel qu'il soit, mais quelques pages plus loin, quand l'amour est le lot de l'héroïne, sa compassion pour les femmes amoureuses ne change pas. Son attitude généreuse d'homme résulte de ses expériences en tant que femme : «Frédéric, connaissant les chagrins d'une tendresse mal reconnue, ne les voulait pas faire souffrir aux autres » (85). Avec le temps Frédéric devient de moins en moins sensible aux malheurs des femmes qui l'aiment et, on le verra plus loin, finit par changer complètement son attitude.

Paradoxalement la générosité voudrait qu'il mente à Yolande, amante sincère et fidèle, et il le fait effectivement. Le mensonge permet d'exprimer les véritables sentiments de Frédéric - femme, mais déguisés en sentiments de Frédéric homme qui, du coup, deviennent faux: «Frédéric, en la trompant agréablement, et voulant bien donner l'essor à la passion qu'il ressentait, lui répondit: «Oui, ma chère Yolande, j'ai été tourmenté depuis votre absence par tout ce que l'amour a de plus cruel, et je n'ai connu la douleur qu'après avoir été privé de la douceur de vous voir» (85-86). En quelque sorte Frédéric ne ment pas car ce sont ses sentiments pour Amaldée qui sont ainsi décrits, mais c'est Yolande qui est la dupe du double jeu de Frédéric.

Il est de même pour Camille. Jalouse et malheureuse, car elle se croyait aimée par le prince de Sicile, elle lui montre l'élégie où tous les sentiments amoureux de la princesse déguisée sont exprimés. Amaldée est présent lors de cette scène et Frédéric souhaite faire part de sa passion à son bien-aimé et en même temps est 
obligé de préserver sa fausse identité : «Quelle fut la confusion de Frédéric ! [...] Il fut quelque temps sans avoir la force de se justifier d'une chose trop véritable; enfin il se sauva encore sur l'équivoque : 'Madame, dit-il en s'adressant à Camille, je vous jure encore une fois que tout ce que j'aime est ici’ »(108). Frédéric ne ment pas, mais trompe Camille qui se croit rassurée.

A aucun moment le prince de Sicile ne s'étonne de l'attirance qu'il suscite chez les femmes. Le déguisement l'explique suffisamment et il trouve normal que leurs cœurs soient trompés comme leurs yeux. Mais Amaldée ne bénéficie pas de la même indulgence et Frédéric s'étonne que le prince de Mayorque ne ressente pas l'amour en dépit des apparences. L'amour étant une passion qui survient sans que la raison et la volonté s'en mêlent, Amaldée devrait en être victime : "Hélas ! disait cette princesse, que ne se sent-il forcé de m'aimer, quoi que sa raison lui oppose ? Je sens bien l'aimer sans le consentement de la mienne; mais il en est encore bien loin. Que n'a-t-il les yeux plus pénétrants, et que ne sait-il démêler le cœur d'une amante sous la figure d'un amant? La langueur de mes yeux devrait, ce me semble, lui avoir développé ce mystère, mais les siens ne l'ont pas voulu voir, ou l'ont méprisée en la voyant» (95). C'est avoir des exigences presque surhumaines, mais l'amant parfait était censé accomplir toute sorte d'exploits. Et Amaldée finit par éprouver «ce je ne sais quoi que Frédéric avait trouvé étrange qu'il n'eût pas encore ressenti » (109).

Amaldée a beaucoup de mal à démêler ses sentiments, car jusqu'à la fin du roman il ne sait pas que Frédéric est une jeune fille déguisée. Sa situation est d'autant plus difficile qu'il ressent un attachement pour Frédéric qu'il n'ose pas appeler par son nom tellement ses propres sentiments lui semblent incongrus.

Au début Amaldée n'est pas amoureux et quand il voit le malheur passionnel de sa sœur, il se félicite, en présence de Frédéric, d'avoir le cœur insensible. Frédéric le prend très mal et s'en va fâché. Comme Amaldée n'arrive pas à expliquer raisonnablement sa réaction, il prend l'amour de Frédéric pour de l'antipathie « et ils furent trompés tous deux par des raisons bien contraires » (95).

Peu à peu le trouble gagne Amaldée, car il ne sait pas comment interpréter le comportement très féminin du prince de Sicile qui « le regarde tendrement » (108, 118), évite sa présence, tombe malade, s'évanouit et surtout Amaldée «se sent entraîné, d'ailleurs sans trop connaître ce qui l'entraîne » (109).

Il reste aussi perplexe face aux sentiments qu'il voue à la princesse de Mantoue. Elle vient pour l'épouser et bien entendu elle aussi, tombe amoureuse de Frédéric. Amaldée et la princesse de Mantoue «avaient conçu beaucoup d'estime l'un pour l'autre et rien de plus, et jamais deux cœurs ne furent plus éloignés dans le temps qu'on les voulait unir » (129). Néanmoins Amaldée ressent la jalousie quand il soupçonne Frédéric d'aimer sa fiancée et il ne sait pas comment interpréter ses émotions : "Je suis insensé, ajouta-il; enfin je suis amoureux, puisque je suis jaloux, et je ne peux l'être que de la princesse de Mantoue". Il ne savait pourtant conclure cela de bonne foi, et il était suspendu entre sa raison et ses sentiments, qu'il ne pouvait accorder » (131-132).

L'énigme quant au sexe du personnage permet à la romancière de créer des scènes où la tension dramatique est très forte et les émotions des lecteurs augmentent. Même dans les situations très embarrassantes le mystère de l'identité du héros doit être préservé. Cela arrive notamment quand Amaldée retourne dans son royaume et après avoir embrassé le roi de Sicile veut embrasser le prince de 
Sicile : «Amaldée s'avançait pour embrasser Frédéric quand il recula de quelques pas. Le roi, qui prit garde à cette action et qui ne connaissait pas le cœur de sa fille, lui fit signe de ne point se découvrir et d'embrasser le prince. Alors une grande rougeur lui couvrit le visage, et le prince, qu'un sentiment respectueux retenait déjà, n'avança qu'en tremblant; et si l'on avait pris garde à cet embarras, sans doute on en aurait été surpris » (109). Le lecteur ne saura pas si les deux princes se sont finalement embrassés, mais imagine bien la gêne des personnages et attend peut-être que le mystère soit rompu.

Il l'est à la fin dans les conditions dramatiques : la reine de Mayorque était, elle aussi, amoureuse de Frédéric, le roi espionnait sa femme et quand le prince de Sicile est venu la supplier de le retenir encore prisonnier à Mayorque, le mari jaloux est entré dans la chambre l'épée à la main : «Il en donna un grand coup à Frédéric, qui se trouva le premier sous son bras. [...] Il fallut déshabiller Frédéric [...]. On visita la plaie; mais Amaldée en reçut une dangereuse, quand il vit une gorge admirable teinte en plusieurs endroits d'un sang qui en relevait la blancheur naturelle ! Ceci ne se peut exprimer. L'amour se faisant connaître chez lui dans ce cruel moment, se fit sentir avec toute sa violence » (151).

Avant la scène de la reconnaissance de nombreux fragments jouent sur le mélange du comique et du tragique. Ainsi l'ambiguïté des sentiments rend la scène du combat naval quasiment comique bien que les soldats meurent et que les circonstances ne prêtent pas à sourire. Le combat oppose l'armée du roi de Mayorque dirigée par Amaldée et celle de Sicile conduite, après la mort de l'amiral, par Frédéric. Les deux princes ne songent «qu'à faire leur devoir », mais la tristesse les accable (116-117). Comme les soldats combattent avec furie, Frédéric «suivit je ne sais quel emportement qui le força d'entrer l'épée à la main dans le vaisseau d'Amaldée: c'était plutôt pour lui offrir son cœur que pour percer le sien. Il demeura si éperdu qu'Amaldée eut le loisir de se remettre de sa première émotion, et lui présentant son épée, «Percez, généreux prince, lui dit-il, percez ce perfide cœur qui n'a osé suivre ses mouvements, et qui n'a pu m'empêcher de faire une action indigne de l'estime que j'ai pour vous » (117).

Le rôle de vaillants combattants est tourné en ridicule : le premier meurt d'amour pour son adversaire tandis que l'autre souhaite se faire tuer par celui qu'il est venu attaquer sur l'ordre de son père. Comme si cela ne suffisait pas Frédéric ému recule et serait tombé dans l'eau si Amaldée ne l'avait pas sauvé, «négligeant tout autre soin, il en rendit seulement à la personne qui l'intéressait sous un nom emprunté » (117). Se trouver si près l'un de l'autre, se tenir la main rend les héros heureux et confus. La scène de combat devient une scène d'amour dont le caractère comique résulte justement du déguisement extérieur qui cette fois-ci n'est pas accompagné de la feinte des sentiments.

Le double jeu que Frédéric doit mener conduit parfois à de véritables scènes de comédie et à des qui pro quo. Au début de la seconde partie les trois femmes qui l'aiment à ce moment-là : Yolande, l'amirale et Camille souhaitent le voir et lui donnent toutes rendez-vous au même endroit. Elles s'y rencontrent et elles voient le prince de Sicile s'en fuir discrètement : «Comme chacune d'elles avait paru ne point remarquer le prince, elles crurent toutes en particulier que le rendez-vous n'était que pour elles, et s'applaudissant toutes de la fidélité de Frédéric et de leur adresse à cacher ce qu'elles en pensaient, elles finirent une conversation assez galante, et se retirèrent fort civilement » (107). Comme dans une comédie chacune 
pense être plus rusée que l'autre, chacune croit être aimée et chacune pense que c'est avec elle que Frédéric est de connivence. La conversation «galante » est la preuve pour chacune de sa supériorité par rapport aux rivales.

Frédéric profite adroitement de leur naïveté et de leur jalousie, car elles s'espionnent l'une l'autre et en fin de compte se gênent si efficacement qu'aucune n'obtient de déclaration de sa part. "Ses maîtresses avaient voulu plusieurs fois l'épier dans ses retraites, et elles en avaient toutes été empêchées les unes par les autres; elles se nuisaient toutes mutuellement, et par la conformité de leurs sentiments, elles se rencontraient presque toujours dans les mêmes desseins: ainsi il était délivré par toutes ensemble de chacune d'entre elles en particulier, et il se trouva moins malheureux par la multitude que s'il n'en avait eu qu'une » (112).

Le rôle que la romancière réserve aux femmes les rend ridicules. L'amirale vient même de Sicile à Mayorque déguisée en homme pour chercher l'amour de Frédéric, comme on le sait, jeune fille déguisée en homme ! Elles tombent toutes amoureuses d'un trompeur, elles le harcèlent et ne se découragent pas, elles n'ont aucune fierté et se font des méchancetés pour se nuire mutuellement. Bref, l'amour les aveugle et les rend stupides et en même temps malheureuses, car Frédéric profite de leur attachement et les scrupules qu'il a manifestés au début de ses aventures s'estompent rapidement.

La princesse de Mantoue devient un objet dont Frédéric et Amaldée se servent pour s'éprouver et se provoquer l'un l'autre. Frédéric étant jaloux d'Amaldée rend sa rivale passionnément amoureuse, passe avec elle tout son temps pour empêcher le prince de Mayorque de la voir. Cela met en colère Amaldée qui essaye à son tour d'entraver les projets de Frédéric. Cette situation est très compliquée car «Frédéric avait quatre maîtresses en même temps : il était l'amant de sa rivale, et le rival de son amant» (135). Il n'est donc pas étonnant que l'ambiguïté de rôles entraîne une équivoque morale.

Contrairement à ce qu'on a vu au début du roman, Frédéric ne manifeste que rarement une compassion pour les femmes qui l'aiment et qu'il trompe. Le côté immoral de son comportement n'est pas évoqué par la romancière mais ne peut pas échapper au lecteur. Par contre Frédéric ressent les scrupules typiques d'une femme amoureuse qui n'a pas le droit de montrer ses sentiments car ce n'est pas socialement accepté. "Ce fut le comble de sa honte, et quoique l'habit qu'elle portait la mît à couvert des soupçons qu'on pouvait avoir de la vérité, c'était assez que d'être coupable en elle-même pour croire le paraître aux autres. Car, bien qu'elle songeât quelquefois à se déclarer, ce dessein ne lui durait guère, et sa fierté l'en empêchait assez » (103).

La conscience morale que l'amour rend coupable, qu'il est honteux pour une femme d'aimer et encore plus de le dévoiler reste toujours très forte. Le déguisement ne le fait pas oublier à Frédéric. Le héros qui arrive dans d'autres circonstances à simuler, à se libérer de ses réactions de femme, face aux principes moraux qu'on lui a inculqués, reste fidèle à sa véritable identité.

Un autre jeu concerne l'identité de l'auteur. Le récit est mené à la troisième personne et l'on ne sait pas si celui qui écrit est un homme ou une femme. Brusquement, à plusieurs reprises, l'accord au féminin ne laisse plus de doutes làdessus. Selon Franco Piva ${ }^{6}$, il est difficile de savoir si Catherine Bernard ne sait

${ }^{6}$ F. PIVA (1993 : 56, 83, note 30). 
pas ou ne veut pas garder sa neutralité en tant qu'auteur. Il est néanmoins significatif que le féminin apparaisse seulement dans les phrases à valeur moralisatrice: «On veut être éloquente quand on est un peu revenue de son premier transport, afin de faire du moins partager ses maux à quelqu'un » (87-88), « quand on est heureuse on enferme toute la joie chez soi, et l'on peut bien n'en faire part à personne, mais quand on est affligée, il faut nécessairement se décharger d'une partie de sa douleur en la confiant à quelqu'un » (113), « on rougit toujours d'aimer la première, soit de honte ou de dépit » (122) ou encore « il est difficile de paraître indifférente quand on ne l'est pas » (140).

Parmi tous les romans de Catherine Bernard, Frédéric de Sicile est celui où il y a le plus de maximes, de phrases à valeur sentencieuse glissées un peu partout dans le récit. Sur même pas cent pages il y en a une quarantaine sans compter les poèmes, eux aussi parsemés de ce type d'énoncées. Outre l'emploi de formes grammaticales au féminin, elles se réfèrent quasiment toutes à l'amour présenté du point de vue d'une femme.

Le roman de l'époque veut remplir un rôle éducatif, veut mettre en garde les lectrices et les prévenir des méfaits de la passion. Catherine Bernard ne cache pas cette volonté et en fait part à ses lecteurs dans les préfaces de ses autres romans ${ }^{7}$. Comme son premier ouvrage n'en a pas, cette façon claire de prononcer à haute voix son avis de femme auteur est peut-être un propos délibéré de sa part. Toute équivoque doit être levée si la leçon morale doit être prise au sérieux. Comme dans le récit la romancière fonde l'intrigue sur l'énigme cela constitue la meilleure preuve qu'elle sait manier le doute et que si elle ne le fait pas dans ses sentences, c'est qu'elle souhaite y révéler subtilement son identité.

Les maximes, telles que je viens d'en citer quelques-unes, n'ont aucun double sens et transmettent clairement un enseignement ou une vérité sur l'amour. Elles expliquent mieux les sentiments embrouillés des héros et constituent pour le lecteur une sorte de trêve au milieu des ambiguïtés.

«Il vivait dans une si parfaite intelligence avec Amaldée que sa félicité l'empêchait de songer que, sous le nom d'ami, elle était amante, et que sa fierté était assez mal ménagée; aussi n'y songe-t-on que quand on n'est pas contente de son amant, et le dessein de la conserver ne naît guère dans le plaisir d'un attachement heureux »(120-121). Outre l'ambiguïté concernant les pronoms, la signification du fragment sur «la fierté mal ménagée » est soutenue par cette phrase à valeur générale avec laquelle il est difficile de ne pas être d'accord : une femme heureuse se soucie moins de conserver sa fierté qui lui est d'un grand secours quand l'amant la déçoit.

Le jeu de l'équivoque est présent dans les poèmes ou les chansons que les héros déclament ou chantent. Sur ce point là Catherine Bernard reste aussi fidèle à la tradition baroque qui préconisait le mélange des genres. Il ne faut pas non plus oublier que la romancière est une poétesse reconnue qui a remporté plusieurs fois les prix de poésie de l'Académie Française et des Jeux Floraux de Toulouse ${ }^{8}$. Il n'est donc pas étonnant de voir les héros recourir à cette forme d'expression de leurs sentiments amoureux.

\footnotetext{
${ }^{7}$ F. PIVA $(1993: 177,239)$.

${ }^{8}$ F. PIVA (1999: 321-354).
} 
La forme de la chanson permet à la romancière de faire communiquer ses héros à distance, de créer des situations où la réciprocité ne joue pas : l'un entend le message qu'il croit lui être adressé tandis que l'autre ne sait même pas que quelqu'un l'écoute. Cela permet d'exploiter l'effet de l'écho. Ainsi Camille chante un air sur le cœur d'un innocent qui tombe dans le piège d'un amour rusé et qui finit par se donner en entier à lui. A ce moment-là l'écho lui apporte la chanson de Frédéric qui n'a pas entendu la sienne :

«Quand d'une vive ardeur on se sent l'âme atteinte

Affecter les dehors de la tranquillité

Est une dure et gênante contrainte.

Lorsqu'en d'étroits liens le cœur est arrêté,

C'est trop que de s'ôter encore la liberté

D'ouvrir la bouche à quelque triste plainte » $(84)$.

Camille qui croit que c'est la timidité de Frédéric qui ne lui permet pas de lui parler de ses sentiments lui répond en disant qu'il ne suffit pas de voir « un cœur soupirer » et que le vrai plaisir, c'est de «se l'entendre dire » (84). Bien entendu quand ils se rencontrent, Frédéric dont les paroles s'adressaient à Amaldée et qui n'a pas entendu les chansons de Camille, répond «assez mal» à un «agréable transport » de la princesse de Mayorque.

Un peu plus tard Camille et Frédéric écrivent des quatrains sur les tablettes. Camille avoue dans le sien qu'auprès « de ce qu'on aime » on oublie «celui que l'on ne sut aimer ». Frédéric lui répond par les vers que Camille interprète comme une déclaration d'amour et la volonté de s'unir plus fortement avec elle. Si elle connaissait le secret de Frédéric elle aurait compris l'équivoque et l'impossibilité de se faire aimer par le prince de Sicile :

«Vous soupirez, hélas ! mais ma peine est extrême,

Mon cœur veut plus pour se charmer.

En vous je vois les yeux, les traits de ce que j'aime,

Mais est-ce assez pour qui sait bien aimer ?» (96).

Sa joie est très brève car Frédéric fait tomber un autre poème de sa poche. Camille y trouve une élégie qui raconte tous les dilemmes passionnels du prince et qui est remplie d'aveux amoureux s'adressant à une mystérieuse personne, «A... ». Même si le poème précédant rassurait la malheureuse amoureuse qui ne pouvait pas comprendre son ambiguïté, l'autre vient immédiatement détruire son espoir et suscite sa curiosité de connaître sa rivale.

Dévorée par la jalousie, elle essaye d'expliquer l'énigme en montrant le poème à toutes les dames de la cour et en examinant leur réaction. Comme par hasard, la troisième amoureuse de Frédéric, l'amirale, porte le prénom d'Amédée et se reconnaît dans l'élégie ! Cela complique encore plus la situation du lecteur qui doit suivre encore une histoire, mais facilite celle de Frédéric qui s'en sort grâce aux intrigues entre les amoureuses.

La fin du roman résout toutes les équivoques. Outre le changement de Frédéric de Sicile en Constance reine de Sicile, Camille découvre que, sauvée du naufrage, elle ne s'est pas trompée de sentiments mais de personne et que c'est le prince Léon et non pas Frédéric qui aurait dû être l'objet de sa passion. Ainsi les deux couples se forment et le poème récité par Amaldée à l'intention de sa bien-aimée rappelle celui que sa sœur avait fait pour Frédéric «dans le temps qu'elle croyait 
être sûre de son cœur » (154) et où seul l'amour partagé et déclaré apporte un vrai bonheur.

Le jeu de l'équivoque est fondé sur le principal procédé du roman baroque, le déguisement de l'héroïne qui n'est pas découvert pendant plusieurs années. Frédéric trompe tout le monde pendant 18 ans et dans des circonstances où il devrait être absolument impossible de sauvegarder le mystère. De ce point de vue le roman n'annonce pas les romans ultérieurs de Catherine Bernard où elle rompt avec cette tradition. En même temps la romancière montre sur l'exemple de l'amirale qui est reconnue très rapidement que le travestissement pour les seuls besoins de l'intrigue amoureuse ne dure pas. D'ailleurs l'amirale est dévoilée car elle est très maladroite et la supériorité de Frédéric en est d'autant mieux soulignée.

Le travestissement de l'héroïne est une tactique complexe et ne constitue pas seulement un ornement qui permet d'élaborer un univers extraordinaire du roman. Le déguisement permet d'avancer l'intrigue, mais il symbolise avant tout le dédoublement de la personnalité de Frédéric qui se cherche soi-même et qui parfois perd conscience de son identité. Frédéric vit mal la situation qui lui est imposée, mais ne se révolte jamais contre son sort, n'évoque ni le mauvais destin, ni la culpabilité de son père qui sacrifie sa fille pour sauvegarder son royaume. La psychologie du personnage, bien que dessinée encore assez sommairement dans Frédéric de Sicile, annonce les romans ultérieurs de Mlle Bernard et leur pessimisme quant à l'amour et à la nature humaine. Lutter pour son bonheur en dépit des contradictions sociales est difficile mais lutter en dépit de ses propres limites et principes devient impossible.

\section{BIBLIOGRAPHIE}

Coulet Henri (sous la dir.) (1992), Les idées sur le roman. Textes critiques sur le roman français $X I I^{e}-X X^{e}$ siècle, Paris, Larousse.

GRANDE Nathalie (1999), Stratégies de romancières. De Clélie à La Princesse de Clèves (1654-1678), Paris, Champion.

GEVREY Françoise (1988), L'illusion et ses procédés, Paris, José Corti.

HIPP Marie-Thérèse (1976), Mythes et réalités. Enquête sur le roman et les Mémoires (1660-1700), Paris, Klincksieck.

Maitre Miriam (1999), Les Précieuses. Naissance des femmes de lettres en France au XVII siècle, Paris, Champion.

PIVA Franco (textes établis, présentés et annotés) (1993), Catherine Bernard Cuvres, tome 1 : Romans et nouvelles, Paris, Schena-Nizet.

PIVA Franco (textes établis, présentés et annotés) (1999), Catherine Bernard Cuvres, tome 2 : Théâtre et poésie, Paris, Schena-Nizet. 


\section{ABSTRACT}

The first juvenile novel by C. Bernard Frédéric de Sicile (1680) is very much different from her later works. The novel is characterised by prevailing baroque style of the beginning of $17^{\text {th }}$ century with less classicistic features. The main theme depicts the process of awakening and a change of identity; from his birth Frédéric must pretend to be a boy instead of a girl, as only a male child can inherit the throne of Sicily. The heroine, by continuous pretending, looses, at times, a track of who she really is.

It is love that forces the disguised girl to make a choice between affection and the duty to dynasty and obedience to her father's will. She tries to show her feelings in a way to prevent mystification to be unveiled. Her lover wavers between hunch and reality. Ambiguity is the basic feature of the novel, at times bringing about comic qui pro quo.

The author manifests her presence in numerous moral maxims and by use of grammatical forms leaving no doubt that the novel was written by a woman.

The work can be interpreted as a search for self-identity while the present pessimistic vision of love and human nature will be a hallmark of C. Bernard's future novels. 